\title{
Internet Governance and Economics of Network Neutrality
}

\author{
Patrick Maillé $^{1}$, Peter Reichl ${ }^{2}$, and Bruno Tuffin ${ }^{3}$ \\ ${ }^{1}$ Télécom Bretagne, Cesson-Sévigné Cedex, France \\ ${ }^{2}$ FTW Telecommunications Research Center Vienna, Austria \\ ${ }^{3}$ INRIA Rennes Bretagne-Atlantique, Campus de Beaulieu, France \\ patrick.mailleatelecom-bretagne.eu, reichl@ftw.at, \\ bruno.tuffindinria.fr
}

\begin{abstract}
This section deals with the network neutrality debate to define the economic relationships between Internet access providers and (distant) content providers. We review the various definitions of what should be a neutral network, the outcome of all international consultations on the topic, and the arguments of network neutrality proponents and opponents.
\end{abstract}

Keywords: Network neutrality, Regulation, Content providers.

\section{Introduction}

The Internet has known an uncontested success during the past two decades, having evolved from an academic network interconnecting researchers who exchange information, to a commercial network accessed by all individuals, made of private access, content, and interconnected transit providers, and over which all type of commerce or content exchange can be performed. Complex relations between those often noncooperative actors have raised discussions about whether or not and how current telecommunications businesses and technological models should be sustained.

A specific question that we want to discuss in this section is the economic relations between Content Providers (CP), i.e., organizations or individuals creating information, educational or entertainment content, and Internet Service Providers (ISP), i.e., the companies or organizations providing access to the network.

In the current Internet business model, ISPs charge both end-users and CPs directly connected to them, the economic relations between interconnected ISPs being generally through public peering or transit agreements. But a key fact is that ISPs do not charge CPs that are associated with other ISPs, and do not treat their traffic in a different way. In addition, there is a specific layers (or Tiers) structure in the relations between ISPs. The so-called «Tier 1 » operators are defined as operators that can reach every other network without any form of fee. Tier 1 operators peer with all other Tier 1 operators. Tier 2 operators on the other hand have to settle peering agreements with other networks, to reach some portions of the Internet. Usually, those operators purchase their traffic to a Tier-1 network rather than discussing with other Tier 2 operators (because it is easier to implement). 
But this business model has been questioned, especially because there is now an important asymmetry in the traffic volume exchanges between ISPs, mainly due to some prominent and resource consuming content providers. The typical example is YouTube (owned by Google), accessed by all users while hosted by a single Tier 1 ISP, and whose traffic constitutes now a non-negligible part of the whole Internet traffic. Moreover, while transit revenues made by ISPs are constantly decreasing, content providers revenues are increasing. As an illustration, it is predicted (by Jupiter Research) for 2012 an amount of $\$ 34.5$ billion in online advertising spending in the USA only, while the transit costs are believed to be under $1 \$$ per Mbps by 2014 . This issue has been the reason why Ed Whitacre, CEO of AT\&T, launched the debate about revenue sharing and the business model of the Internet in 2005 [8]. Basically, Ed Whitacre claimed that distant CPs use the AT\&T network for free in order to reach end users, and that it could not be economically sustained: they should be charged by ISPs they are not directly connected to, especially because of the decreasing transit prices. Indeed, the ISPs do not have any incentive to invest into a network architecture, by improving the actual one or by implementing a new one. Another management possibility to differentiate CPs, not related to charging, has been implemented by the USA provider Comcast in 2007, which blocked P2P applications such as BitTorrent, under the argument that P2P content is mostly illegal.

All this has led to a strong protest from CPs who are losing accessibility or quality of service, and from user associations claiming that it is an infringement of freedom of speech. This raised the so-called Network Neutrality Debate, bringing questions at the same time at the economical, legal, technical, and political levels. The purpose of this section is to review the proposed definitions of network neutrality, a notion actually not formalized, to summarize the outcomes of the international consultations held very recently, and to describe and discuss the arguments of both proponents and opponents of neutrality to allow the reader to make her own conclusions.

It is important to remark that even governments somewhat see the position of CPs as an issue, in accordance with ISPs complaint. In France for instance, there is a worry about the online advertising revenue not being taxed as usually done, and a proposition on that matter has been released (see [13]). Between 10 and 20 million Euros could be collected in taxes from the USA providers such as Google, Microsoft, Yahoo and Facebook.

\section{Network Neutrality Definitions}

Maybe surprisingly, there is no clear and generally admitted definition of network neutrality. A network is often said to be weakly neutral if it prohibits user discrimination [11,12], which means it does not charge users differently for the same service, but can differentiate between applications (example: video having a priority with respect to email because of more stringent QoS requirements). It is said to be strongly neutral if in addition it prohibits any service differentiation that is if it does not allow managing packets differently. 
A tentative of « official » definition of neutrality was made in 2005 in the USA by the Federal Communications Commission (FCC) stating that network neutrality could be summarized into four items [3]: (1) no content access can be denied to users (2) users are free to use the applications of their choice (3) they can also use any type of terminal, provided it does not harm the network (4) they can competitively select the access and content providers of their choice. This corresponds exactly to the wellknown proposition/definition has been described by Professors Timothy Wu and Lawrence Lessig; summarized by Tim Wu, "Network neutrality is best defined as a network design principle. The idea is that a maximally useful public information network aspires to treat all content, sites, and platforms equally."

\section{International Consultations}

The debate has been so strong, and the potential impact of a regulation or nonregulation so important that governments have held public consultations. This has started in the USA, but and also recently in France (by ARCEP, the French regulator), UK (by Ofcom) and by the European Commission (EC) in 2010, after Norway (by the Post and Telecommunications Authority, NPT) and Sweden (by the post and Telecom Agency, PTS) in 2009. In that same year, the FCC has imposed neutrality regulatory rules, a decision that has been appealed by an operator, Verizon. The goal of those consultations was to (re)design an Internet that suits the interests of all actors (consumers, broadband service providers, entrepreneurs, investors, and businesses of all sizes) thanks to the most appropriate trade-off. Even if everyone was allowed to respond to the consultation, it has mainly been answered by specialists such as computer scientists, industry lobbies and non-governmental organizations. The participation was different across the countries, and when individuals were responding, they were largely defending neutrality.

One of the most important outcomes/recommendations is that traffic management has to be transparent, in the sense that operators have to clearly explain what they are implementing. This is also considered as being a manner to avoid anti-competitive behaviors from operators and to control if a minimal best-effort Internet is in place. Transparency has been a consensus among all industrial telecommunication actors. ARCEP proposes that operators provide all clear and relevant information on access, QoS, and limitations (if any) [1,10].

Another key idea is to protect customers' rights to access content, which cannot be forbidden, i.e., the network should be open, which is defined as allowing "end users in general to access and distribute information or run applications of their choice" [2].

Another aspect is quality of service (QoS). A result of consultations is the demand to define a minimum level of QoS (which has to be defined), for a type of universal access principle. Weak neutrality and service degradation would be allowed if such a minimum level is reached. No regulator seems to have something against discrimination if a minimum QoS is guaranteed. It is even considered that a total lack of traffic management could result in a lower perceived QoS for users. In this sense, being 
neutral and open does not necessarily mean that all bits have to be considered equal. The FCC advocates for a "reasonable network management" vaguely defined as " (a) reasonable practices employed by a provider of broadband Internet access service to (i) reduce or mitigate the effects of congestion on its network or to address quality-ofservice concerns; (ii) address traffic that is unwanted by users or harmful; (iii) prevent the transfer of unlawful content; or (iv) prevent the unlawful transfer of content; and (b) other reasonable network management practices" [4]. This could be realized as soon as transparency is fulfilled.

All those aspects mean that the Internet needs to be supervised and monitored by regulators to verify if the above proposed rules are satisfied. It is a proposition for and from all regulators, even outside the European Union. In addition, it is asked in the $\mathrm{EU}$ (where the EC is entitled to impose rules) to forbid operators to block users from accessing legal content. ARCEP has been more stringent, asking for nondiscrimination between Internet traffic streams [1].

A last recommendation is that neutrality requirements should be different depending on whether we consider mobile or fixed networks, because (1) capacity is much more limited in the mobile case, (2) because of mobility itself, which has to be handled by operators, and (3) because of the larger type of attached devices (phones, smart-phones, netbooks, broadband cards), complicating the management. Traffic management has, therefore, to be more flexible in the case of mobile networks, due to their specificities [1].

The impact of the decisions made after those consultations is of great importance at the global political level too. Indeed, as highlighted by FCC Commissioner's Robert M. McDowell [5], several countries were waiting for the USA (and E.U.), and some regulatory decisions could be used to justify and expand their own government authority over the Internet, but with the motivation to limit the freedom of speech. This has to be taken into account when taking decisions.

\section{Arguments of Neutrality Proponents: An Idealistic and Humanist View}

The Internet architecture is based on the notions of layers, as defined by the Open Systems Interconnection model (OSI model). Almost all layers are managed by independent entities (or providers). The OSI model abstracts the network into seven layers (physical, data link, network, transport, session, presentation, application) and each entity at a given level interacts only with the layer beneath it, and provides "capacities" to the layer above it, the relations between layers being operated thanks to standardized protocols. In the real Internet, this model can be simplified into a model made of four layers [6, 7]: the content layer (web pages, emails, telephone calls, video, etc.), the application layer (the software permitting the content to be developed), the logical layer (TCP/IP, domain names, all the protocols), and the physical layer (cables, routers, servers, wireless base stations). If there is no neutrality, there is a major risk that this successful and cheap model will be destroyed because ISPs may 
reduce competition by developing exclusivity arrangements with content and application providers, and use proprietary protocols between layers that may harm the development of new applications by third parties. Changing the structure and the way the Internet has been designed can be dangerously harmful. Without neutrality; it is claimed that the last mile ISPs, who have the market power, will discriminate between content providers, favoring the ones that are connected to them, so that end users will have less choice, which is claimed to be economically inefficient (what economists contest, see the next subsection). This market power (whoever is the most powerful) is said to be a reason to introduce regulation to maintain innovation at the content level. Similarly, having an important level of innovation from CPs will foster access demand of end users and therefore increase or maintain revenues of ISPs.

A main principle attached to the networking (disaggregated) layer architecture, and described as a key factor, is that the network can hardly make a differentiation according to the identity. This is related to an important issue developed by the neutrality proponents: the freedom of speech on the Internet [7, 8]. If the network is not open, this simplifies a lot its control by governments or political entities willing to limit information diffusion and retrieving the identity of correspondents. The current layered architecture on the other hand allows a relative anonymity, a decentralized distribution (which makes it easy and cheap to implement), the possibility to reach multiple access points, no simple system to identify content, and tools of encryption; this is exactly what is needed by political parties to speak up in an oppressive country. This political aspect is hardly taken into account by neutrality opponents and economists, but we can note that the current infrastructure has very probably helped the recent Arabic revolutions with all the information exchanged. Changing it could harm the development of democratic values worldwide.

In general also (not only talking about freedom of speech), vertical integration of content and access, as aimed by non-neutrality proponents, especially in an exclusive way, is seen as discriminatory by users and by regulators. Considering other communication areas which have a longer history, such as roads, canals, mail, etc., there has always been a requirement of interconnection and to serve the public, even if resources are owned by private companies.

And the argument of ISPs that revenues decrease and investments are not possible anymore are contested because ISPs keep those cost values secret; if they were more transparent, data could be verified and they could be trusted. It is rather seen that ISPs want to earn money from both ends: end users and CPs.

Finally, a last important reason for neutrality is universality (as above on international consultations): all consumers are entitled to reach meaningful content, whatever the technical limitations of their service. They are also entitled to attach any type of device to the network; and to run any application. The basic idea behind that principle is to keep all the population informed and able to communicate, and not to increase the gap between rich and poor people. French government for instance is currently trying to set up an access for the poor at a limited price. 


\section{$5 \quad$ Arguments of Neutrality Opponents: An Economic View}

As presented in the introduction, the initial reason of ISPs to deviate from a neutral behavior is the disincentive to invest in networking capacities because of the reduced revenues, an issue argued to be often forgotten by neutrality defenders who always seem to focus on CPs innovation only.

The most important argument is that vertical integration, which consists in bundling content and access into a single service, and which can be exclusive for some type of content, is often shown to be the most efficient economically. It has indeed been demonstrated by the Chicago school of antitrust analysis that vertical arrangements can even be efficient under a monopoly, because a monopolist can gain from a higher total value of the whole platform (made of access plus content). A typical example, in another but related context, is Microsoft that, while in a quasi-monopole on the operating systems of computers, gains from the development of applications because it increases its own value (the equivalent of Microsoft being here either the ISP or the $\mathrm{CP}$ ). The Chicago school of antitrust analysis has more exactly proved that there are preconditions under which vertical integration might be harmful: 1) the vertically integrated firm must have market power in its market (to be able to use this powerful position), and 2) the market in which the considered provider will be integrated must be concentrated and protected by barriers to entry [7]. It is claimed that none of those preconditions are met in the current telecommunication industry, and therefore vertical integration would be more efficient than keeping the current neutral model. Vertical integration has for example been successful in cable television in the 80 s in the USA, with cable operators investing a lot in the network to be able to respond to demand for content.

In addition, the Internet is rather seen now as a media (i.e., content distribution) business, and should then be treated as such rather than as a classical telephony/telecommunication economic business facility. Indeed, users go on the Internet not for broadband in itself, which is rather a mean, but for the content, goods and services that they can find. As a consequence, vertical integration is therefore more relevant here because in such media business, distribution (broadband access for us) is generally bundled with content. We can remark that it is already implemented, even if in a limited way: almost all ISPs provide a portal over which users can access a predefined content: (selected) news, direct access to some content, etc. This does not prevent (yet) the users from accessing the content of their choice, but is an incentive to go to the one preferred by the ISP.

But vertical integration is not a new topic, it has always been in discussion in the Internet industry, and is not a new issue. Notable examples are when Microsoft incorporated its web browser into its operating system, introducing a major debate, or when AOL (an ISP) and Time Warner (a CP) merged.

It is even advocated that, contrary to the intuition, vertical integration and nonneutrality would enforce competition at the network (ISP) level [7]. This is for instance what happened between cable and DSL providers in the USA, who drastically decreased their price because of the competition, at the benefice of end users; they also may fight on content. As an illustration, we can cite the competition between 
cable and satellite TV in the USA: (satellite) DirecTV managed to get an exclusivity on the Sunday night NFL game, which could then not be accessed by subscribers of cable operators, except if they paid in addition for the satellite operator. This put pressure on the historic cable operators to improve their content, to innovate in this direction and to develop competition. A neutral network would not have permitted the development of DirecTV. In addition, it is anyway unlikely that ISPs would decide to cut the access to major CPs, such as Amazon, Facebook, etc., because they would immediately face protests and pressure from their subscribers.

Similarly, allowing the ISPs to differentiate service would increase the economic efficiency and social welfare, by responding to the heterogeneity of users in terms of preferences. This means applying revenue management techniques [9] to telecommunications as it has been done in the airline industry, hostelling, etc., with a success that has not been discussed in those areas. Users would benefit from this, since they would be able to choose the offer most adapted to their preferences, and it may even drive to more services attuned to segments of the population. It is even claimed that the introduction of regulation could favor some applications against others, an undesirable effect. Also, differentiation and market segmentation would allow many ISPs to survive, since if not implemented, declining costs otherwise usually result in a limited amount of surviving companies and natural monopolies. As a last remark on service differentiation, we can note that the network "naturally" makes some differentiations anyway: a protocol like TCP gives less throughput to users far away, and BGP allows to discriminate at routers.

Opponents of neutrality, even if they admit that the success of the Internet is due to the end-to-end architecture, believe that it is not a reason for not changing it now: it has the right to evolve because of economical changes. Even more, writing in the stone a rule because it initially worked might be really harmful. It is not because the architecture has been optimal that it will continue to be. They rather see this immobility reaction as a scare of change from computer scientists, not ready to lose the "baby" they gave birth to, but the Internet users are in general not highly skilled (in opposition to early subscribers to the network) and are not that sensitive to this issue. As mentioned by A. Thierer [7, Page 94]: "If consumers really wanted a pure dumb Net connection, then why does AOL's walled garden have over 30 million subscribers worldwide while charging $\$ 23.90$ per month?”. We do not know exactly what will be the next business model, but there is no reason to necessarily keep the one in place.

Even at the CP level, vertical integration is said to be preferable because it may help (especially in a segmented market) to push applications that have a limited impact. Using proprietary protocols for development and distribution could simplify the advent of new applications, and thus foster innovation thanks to a larger range of networking tools. And as the above example on the NFL games shows, competitive ISPs have an incentive to help content developers in a non-neutral regime, particularly when there is a monopoly for some type of content. Interoperability, even if appealing a priori, is not necessarily efficient from an economic point of view.

Promoting a universal access to the network is also contested by neutrality opponents. They believe that this may harm investment because there is no real incentive in that situation for ISPs to invest in capacity and technologies if access is granted, 
this even more if service differentiation is not permitted: how to define access prices if access is mandatory? This universal access could maybe be discussed for applications such as web browsing or email, but not for bandwidth-consuming applications. In addition, it is argued that the full connectivity as a principle to maximize social welfare does not take into account an important component: the congestion that end users will experience if there are no sufficient investments on capacities or technologies by ISPs.

This universality principle has been somewhat questioned by governments too. As an illustration, France has passed a law in 2009 that disconnects users from the Internet if they download illegally copyrighted material. Even if this has raised a debate at the law level (the French Constitutional Council asked to modify its implementation because it was in contradiction with the Declaration of the Rights of Man and of the Citizen coming from the French revolution: the ISPs will not decide by themselves to filter traffic, but will implement the decision if it comes from a judicial authority), such a law has been enforced (the so-called HADOPI law), even if the Internet has been seen as a mean to exercise the freedom of speech. This differentiates the treatment of legal and illegal traffic, but the notion of legality being considered differently in other countries, this could go against freedom of speech.

It is also remarked by neutrality opponents that imposing a regulation on neutrality would just add or shift power (even) closer to CPs, especially in the case where there is a limited competition. Typically, Google would become even more powerful with YouTube that already accounts for an important part of the whole Internet traffic.

Even at the security level, a vertically integrated service is advocated to ease the control of attacks (because the current model is more or less based on trust and cooperation between communicating nodes) and limit illegal content, something of interest for regulators.

\section{Conclusions}

As sketched by our presentation, the network neutrality debate can rather be seen as the opposition between (1) an idealistic (neutral or weakly neutral) network as imagined initially by scientists, with an organization in layers, a low cost, and for which end-to-end connectivity and universality are the key issues, and (2) a purely economic (non-neutral) view of the network. The goals of both worlds are therefore different and make a strict comparison difficult.

A question is then: are end-users and computer scientists just scared of the potential change? Or is it too risky and could it potentially lead to a "telecommunication / connectivity" crisis? Would it be accepted by the public as easily as it accepted the Internet in its current form? We wish the reader to make her own opinion on the subject and hope that our discussion will be helpful towards that goal.

We may mention a particular case where neutral and non-neutral networks coexist: the road network, where you have commercial (highway) roads with tolls, and national roads, with a lower quality of service, but that are free of charge and allow (in most countries) a full connectivity. Those national roads are maintained by states, and one could check how this (economic) model could be transferred to telecommunications. 
An important remark to conclude this section is about another neutrality threat, that could be viewed as the most "dangerous" and that is not mentioned enough, the search engine neutrality. To illustrate the problem, Google's CEO, Eric Schmidt, actually had to testify in September 2011 before the US Senate about a possible discrimination in the ranking of the searches on the engine, because it is claimed by some to favor the content provided by Google itself. Typically, but not only, favoring YouTube in front of competitors such as Dailymotion. Search engines are indeed very important actors in the current Internet, being the mean by which most content is reached. Their activity can greatly influence the way the network is used and their behavior has to be carefully reviewed too. Because of that, Google is facing a risk to be dismantled under the antitrust law.

Open Access. This article is distributed under the terms of the Creative Commons Attribution Noncommercial License which permits any noncommercial use, distribution, and reproduction in any medium, provided the original author(s) and source are credited.

\section{References}

1. ARCEP: Neutralité de l'internet et des réseaux: propositions et orientations (2010), http: / /www . arcep.fr/uploads/tx_gspublication/ net-neutralite-orientations-sept2010.pdf

2. Commission of the European Communities: Telecom reform 2009: Commission declaration on net neutrality. Official Journal of the European Communities L337 (2009)

3. Federal Communications Commission: Appropriate framework for broadband access to the Internet over wire-line facilities. FCC 05-151, CC Docket No. 02-33 (September 23, 2005)

4. Federal Communications Commission: Notice of proposed rulemaking. FCC 09-93 (October 2009)

5. Federal Communications Commission: Order in the matter of preserving the open internet, broadband industry practices. FCC (April 2010)

6. Lemley, M., Lessig, L.: The end of end-to-end: Preserving the architecture of the internet in the broadband era. 48 UCLA Law Review 4 (2001)

7. Lenard, T., May, R.E. (eds.): Net Neutrality or Net Neutering: Should Broadband Internet Services be Regulated? Springer (2006)

8. Odlyzko, A.: Network neutrality, search neutrality, and the never-ending conflict between efficiency and fairness in markets. Review of Network Economics 8(1), 40-60 (2009)

9. Talluri, K., van Ryzin, G.: The Theory and Practice of Revenue Management. Springer (2004)

10. Wong, S., Rojas-Mora, J., Altman, E.: Public consultations on Net Neutrality 2010: USA, EU and France. Tech. rep., SSRN (2010), http: //papers.ssrn.com/sol3/papers.cfm?abstract_id=1684086

11. Wu, T.: Network neutrality, broadband discrimination. Journal of Telecommunications and High Technology 2, 141-179 (2003)

12. Wu, T.: The broadband debate: A user's guide. Journal of Telecommunications and High Technology 3, 69-96 (2004)

13. Zelnik, P., Toubon, J., Cerutti, G.: Création et internet. Ministère de la Culture et de la Communication, République Française (2010), http://www.culture.gouv.fr/ mcc/Espace-Presse/Dossiers-de-presse/ 